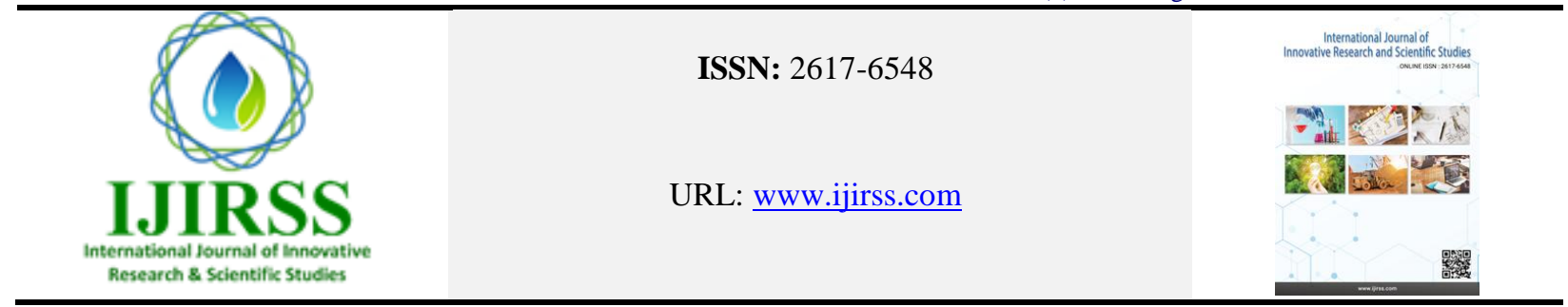

\title{
An Overview of the Establishment and Strength of the Shir and Khurshid of Timurid Governance System
}

\author{
Mahdi Mysam \\ ${ }^{1}$ Education Faculty, Farah Institute of Higher Education, Farah, Afghanistan \\ (Email: mysammahdi59@gmail)
}

\begin{abstract}
Following the Mongol era and the instability of the llkhanis, Amo river areas and Transoxiana saw a favorable situation to turn into a great empire. Amir Timur Gorkani who had established himself as a person with great military intelligence in Transoxiana, gathered his loyal forces and with his resolution and valor founded a government that made many people from east to the west fear even by hearing his name. Timurid banners were designed with pictures of a lion and the sun that were associated with tyranny and light. This government unique characteristics had enabled it to stabilize its dominance over a great area after a short period of time. In order to recognize Timurian strong foundation, its governance must be studied. In the scope of research purposes, library methods and analyzing authentic sources of timurd authors have been used. Military arrangements based on Genghis Khan's yasai and use of public fear for conquering different areas, an economical - governmental system compatible with the situation of the time, appointing competent and reliable people for governmental posts and integrating a Mongol and Islamic government in itself answered all governmental necessities. Of course, Timur was a Muslim himself but one of those Muslims who allotted most of his time for life and governance. Forced immigration of artesian to the government's favored areas made Samarkand and Bukhara to be considered among the best cities of the world. In this work it has been tried to study Amir Timur's governance for political, economic and military stability using authentic academic resources.
\end{abstract}

Keywords: Yasa, Timurid, Seorghal, Eqta, Shir and Khurshid.

DOI: $10.53894 /$ ijirss.v2i3.18

Funding: This study received no specific financial support.

History: Received: 3 May 2019/Revised: 17 May 2019/Accepted: 1 July 2019/Published: 7 July 2019

Licensed: This work is licensed under a Creative Commons Attribution 4.0 License $(\text { (c) })_{\text {EY }}$

Competing Interests: The author declares that there are no conflicts of interests regarding the publication of this paper.

Transparency: The author confirms that the manuscript is an honest, accurate, and transparent account of the study was reported;

that no vital features of the study have been omitted; and that any discrepancies from the study as planned have been explained.

Ethical: This study follows all ethical practices during writing. 


\title{
نكرشى بر تأسيس و استحكام نظام كشوردارى 》شير و خورشيد) تيموريان
}

\author{
مهدى ميثم \\ دانشكدة تعليم و تربيه، مؤسسةُ تحصيلات عالى فراه، فراه، افغانستان
}

\begin{abstract}
خلاصه

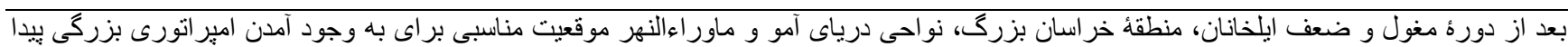

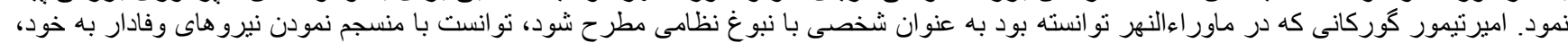

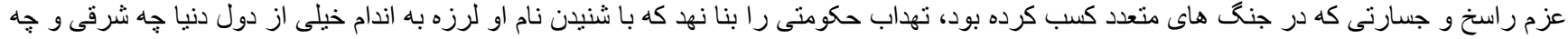

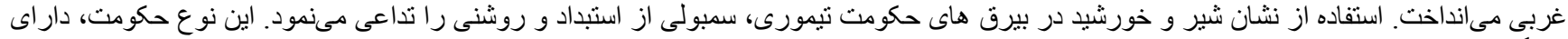

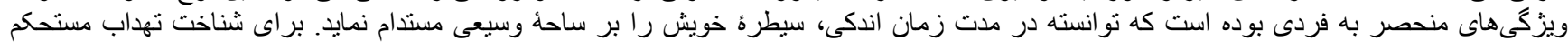

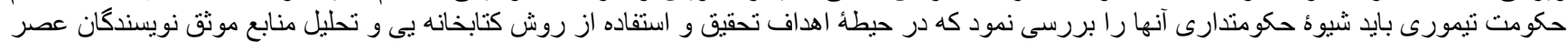

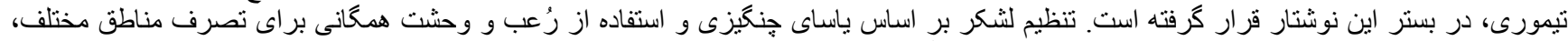

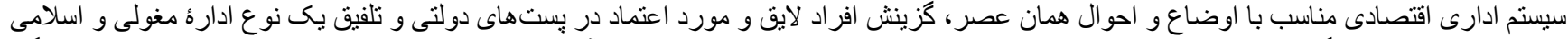

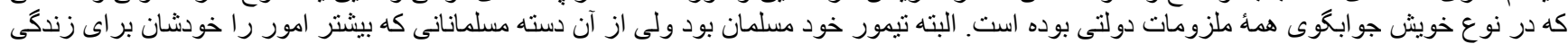

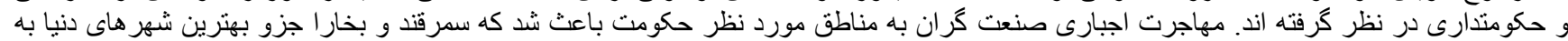

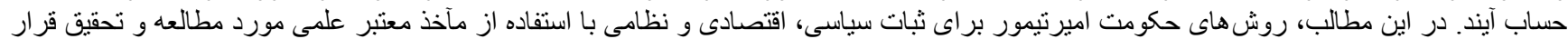
كرفته است.

كلمات كليبى: ياسا، تزوكات، سيورغال، اقطاع، شير و خورشيد.

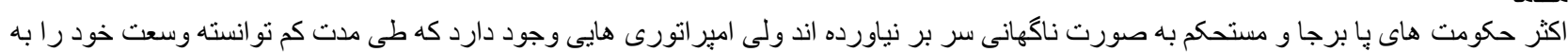

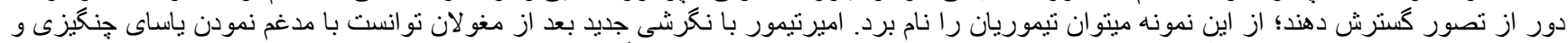

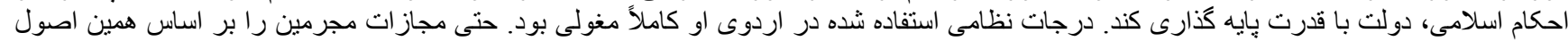

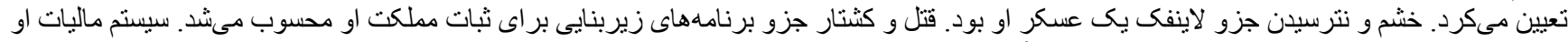

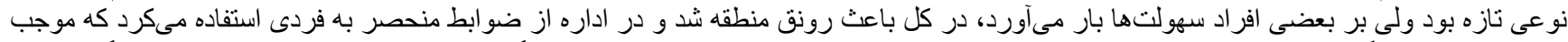

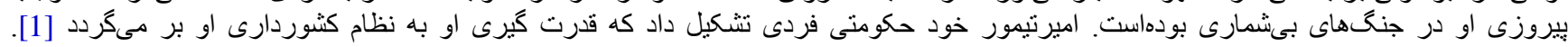

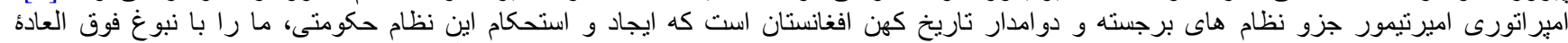

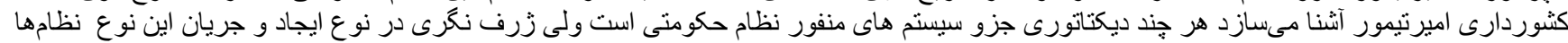

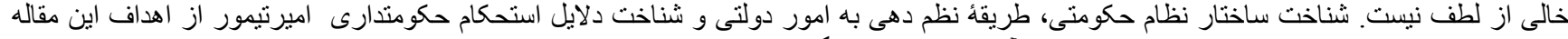

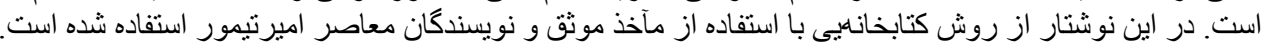

تشكيل حكومت تيمورى مئر

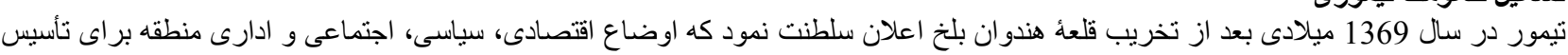

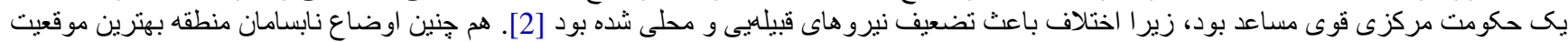

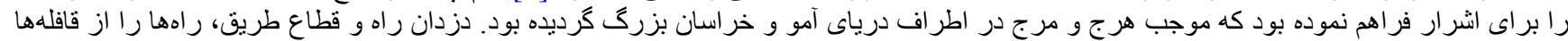

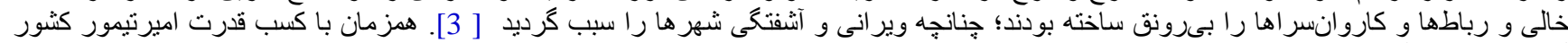

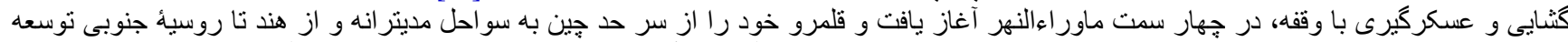

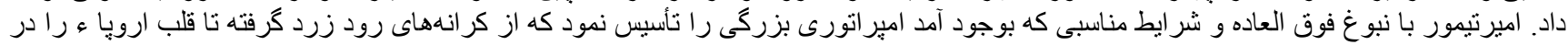

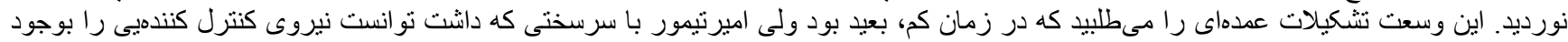

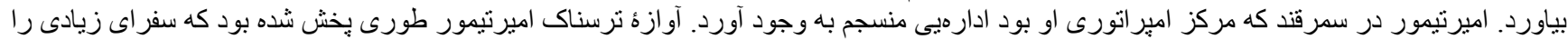

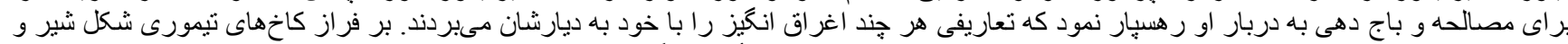

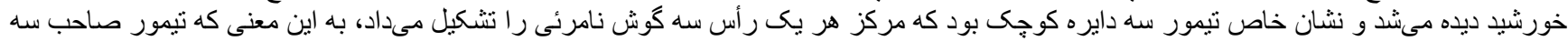

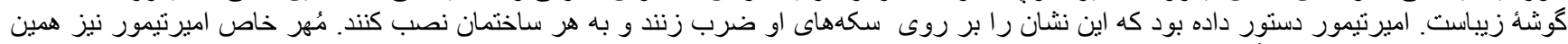

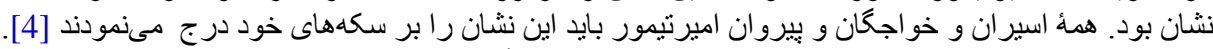

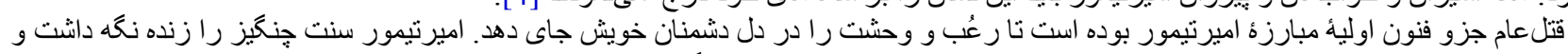

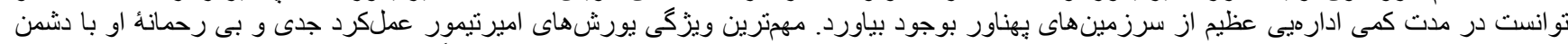

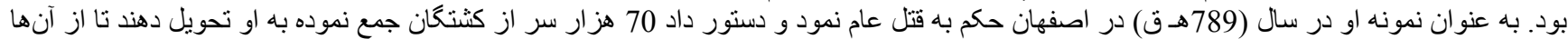

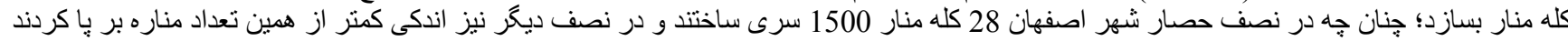

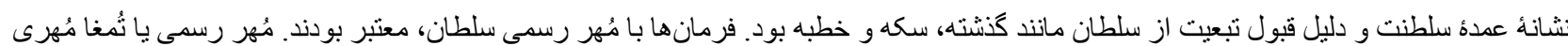

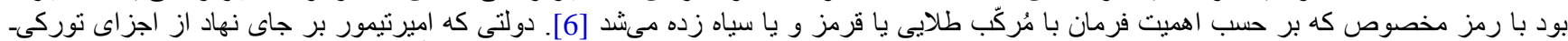

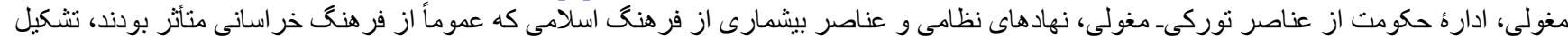

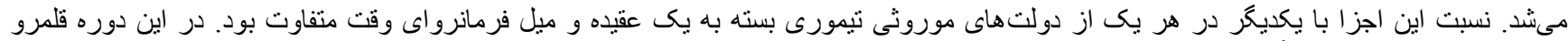

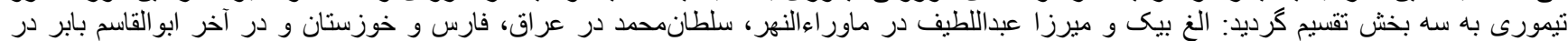

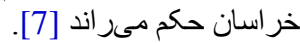

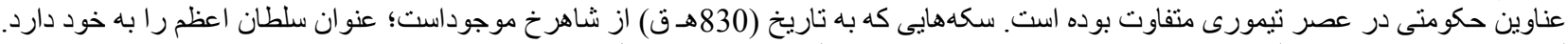

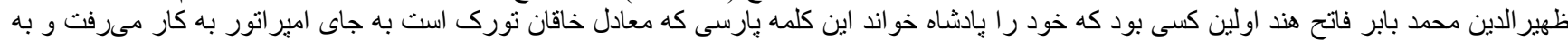

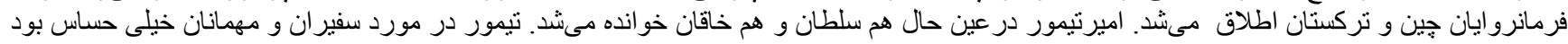




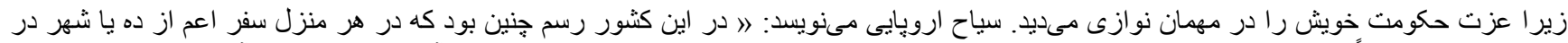

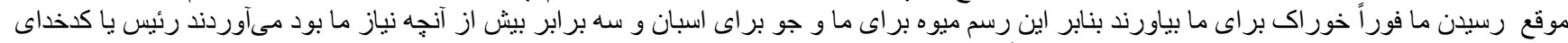

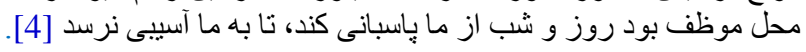

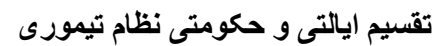

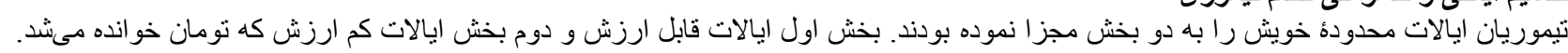

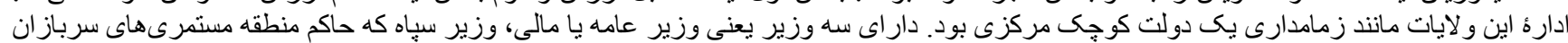

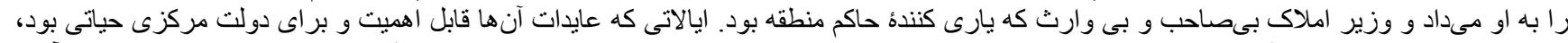

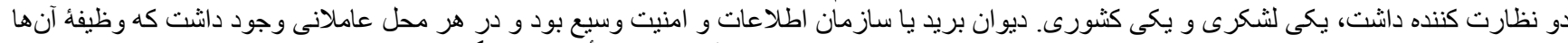

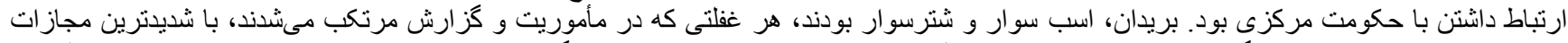

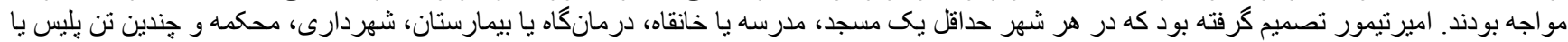

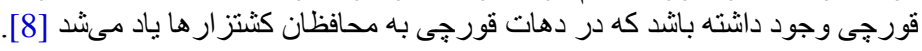

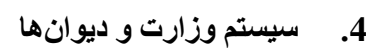

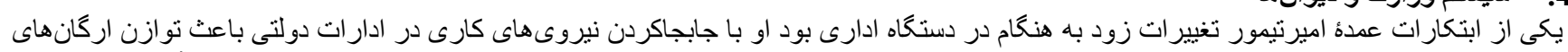

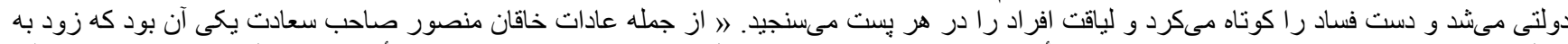

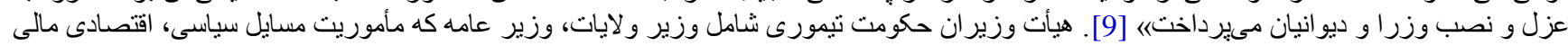

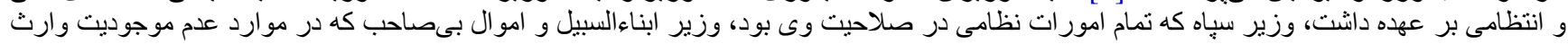

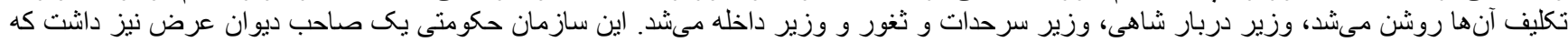

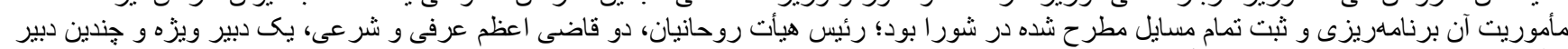

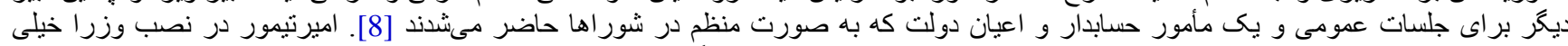

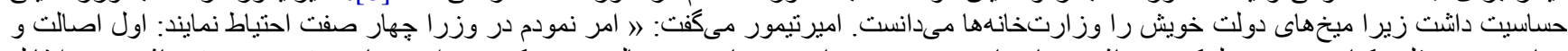

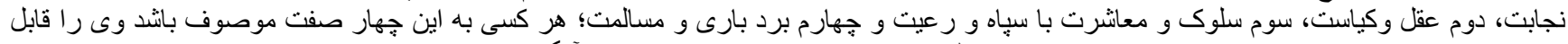

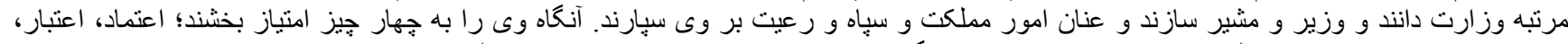

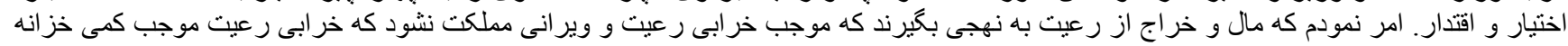

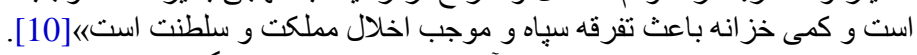

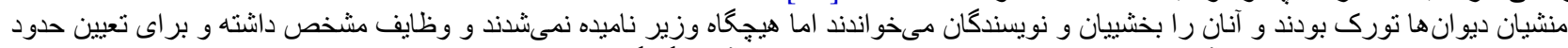

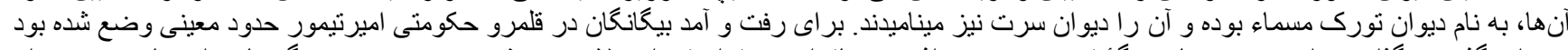

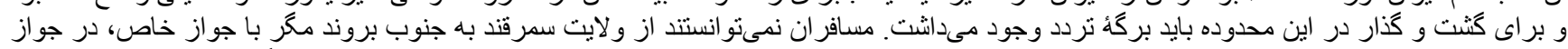

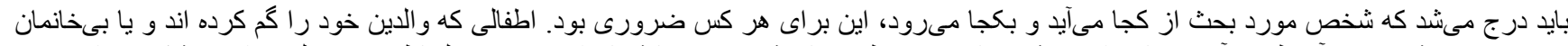

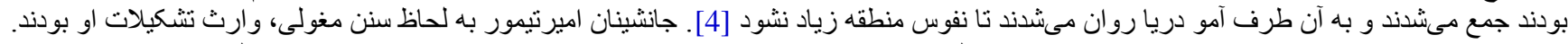

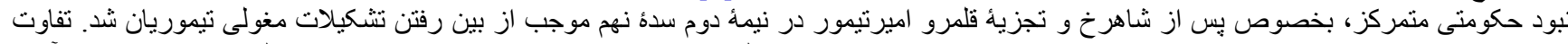

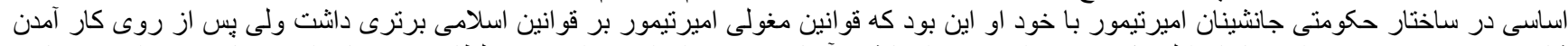

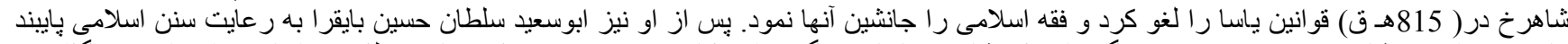

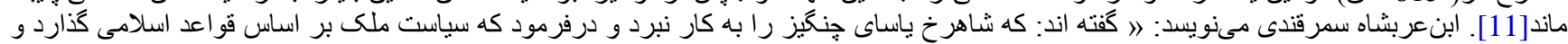

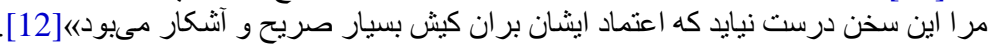

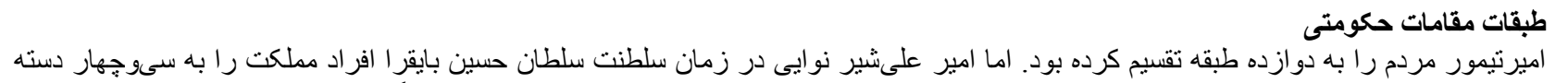

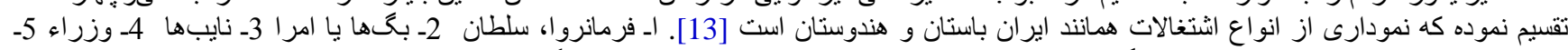

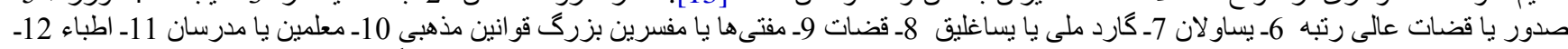

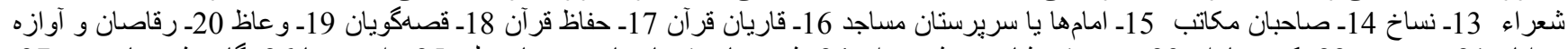

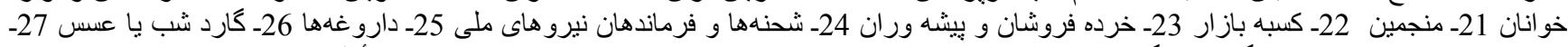

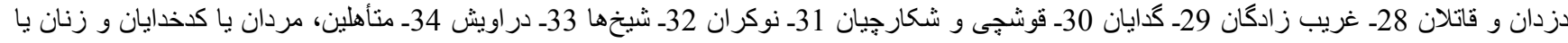

كدبانو [8]

\section{تزوكات تيمورى}

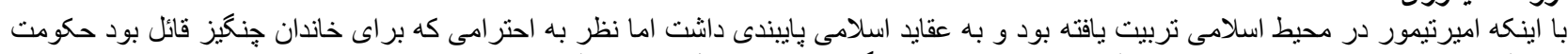

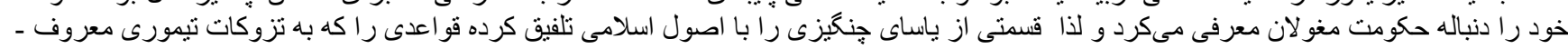

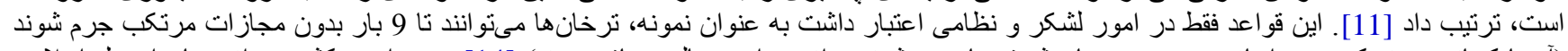

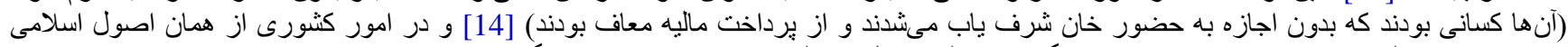

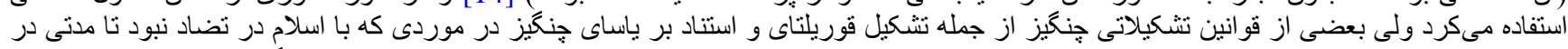

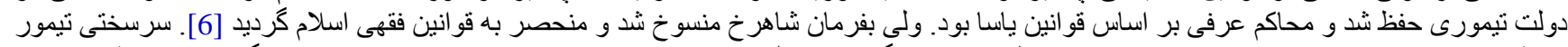

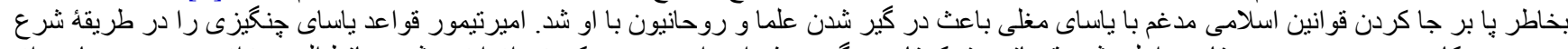

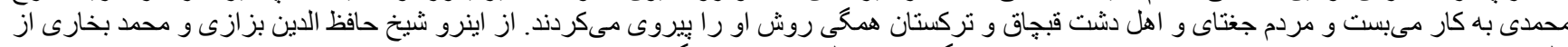

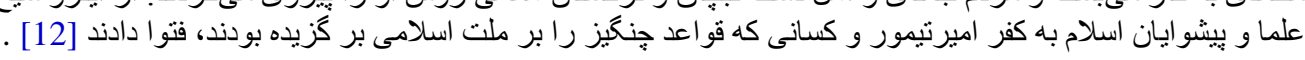

قضاوت

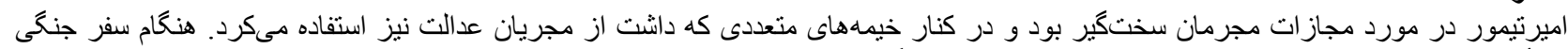

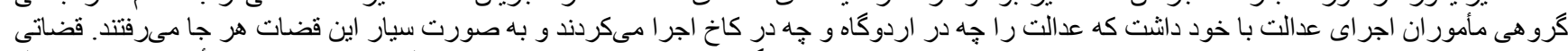

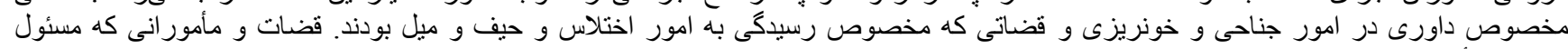

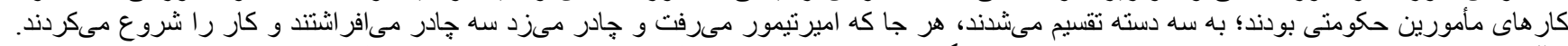

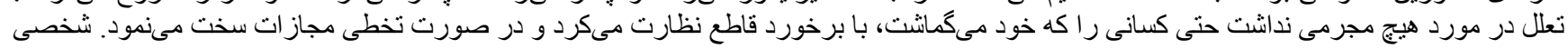




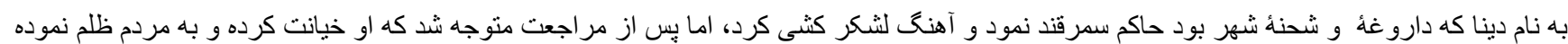

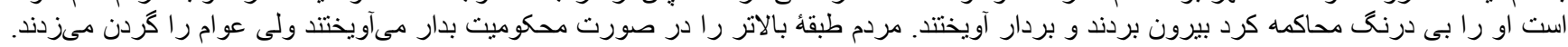

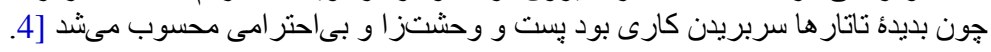

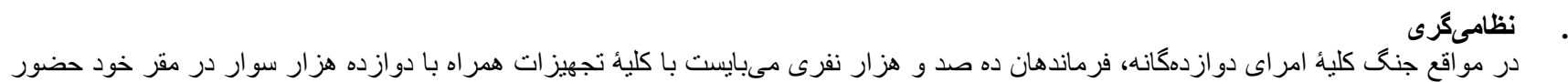

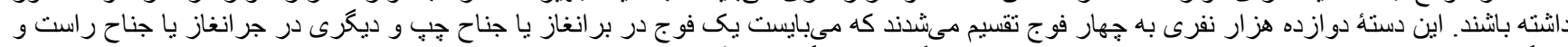

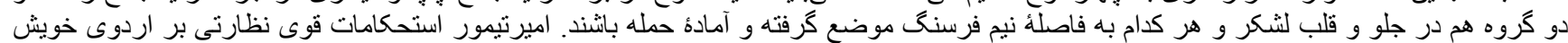

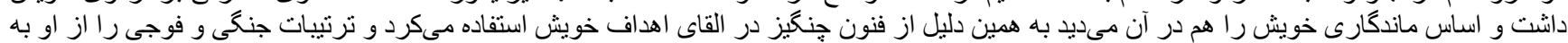

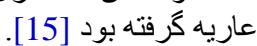

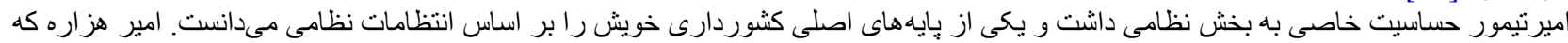

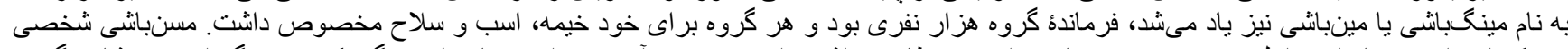

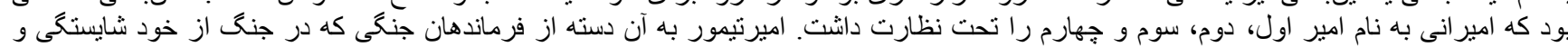

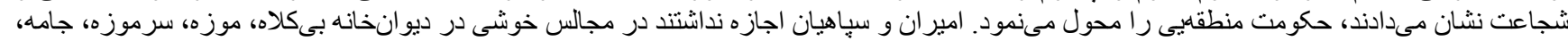

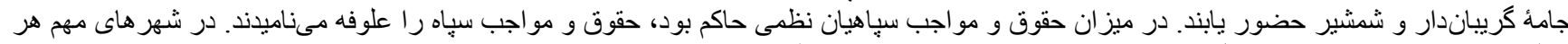

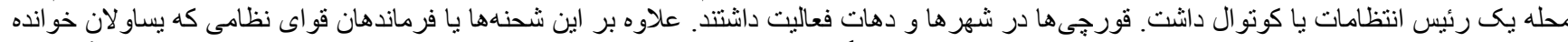

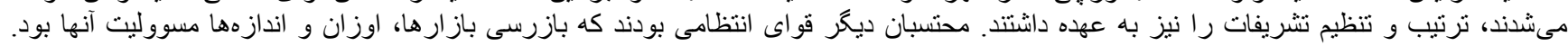

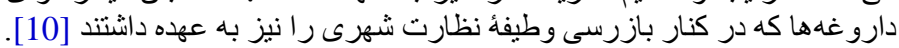

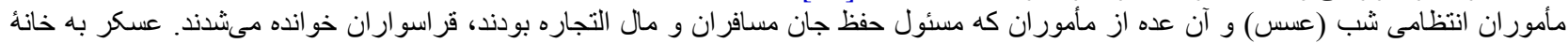

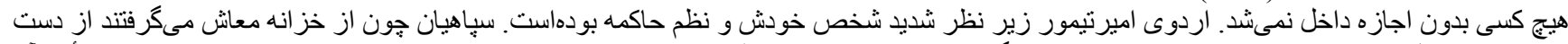

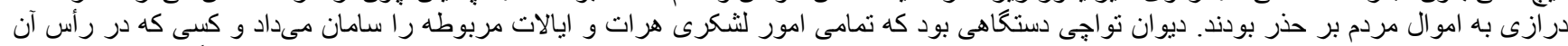

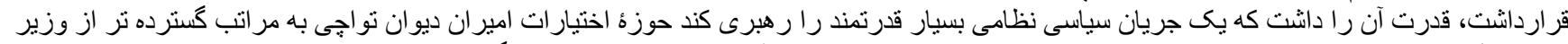

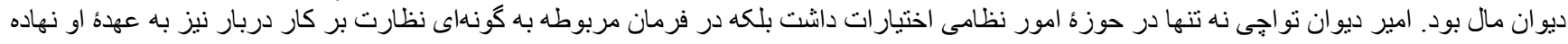

شدمبود [16] مال بود.

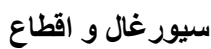

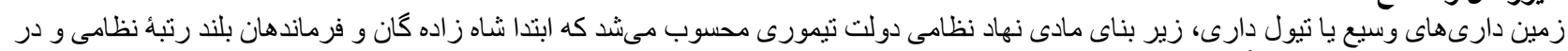

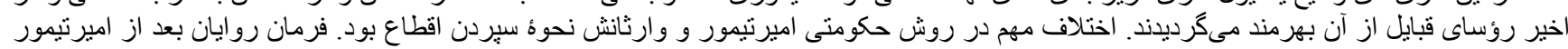

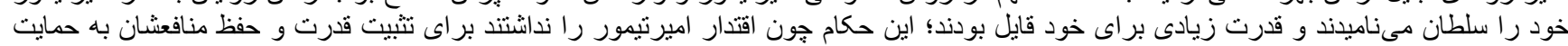

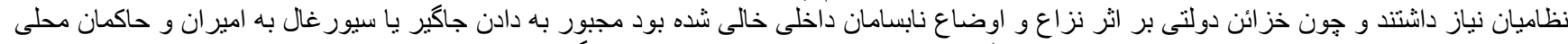

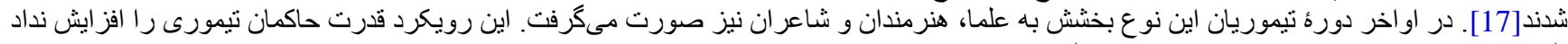

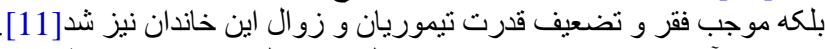

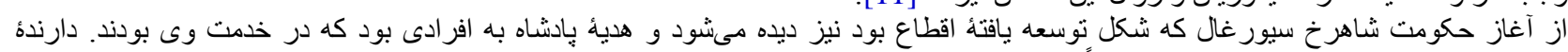

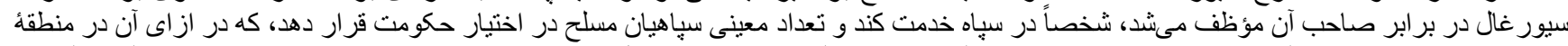

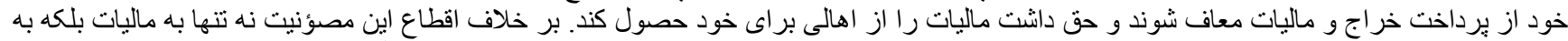

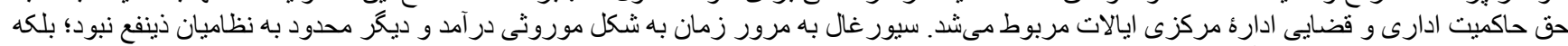

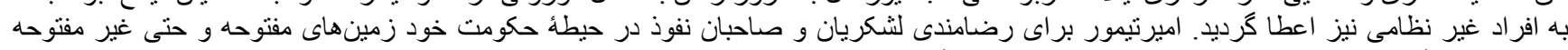

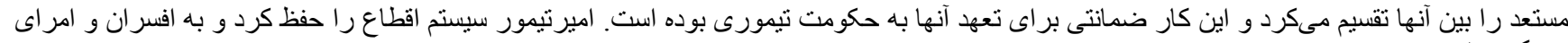

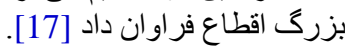

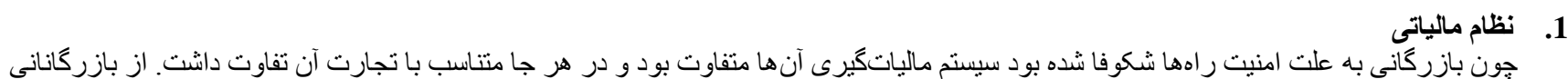

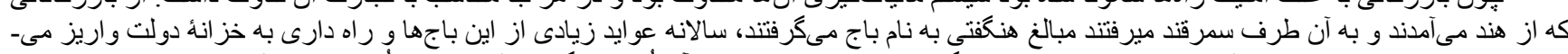

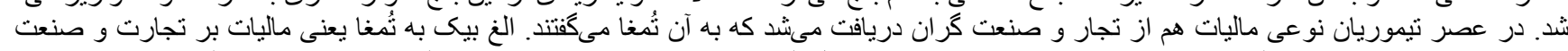

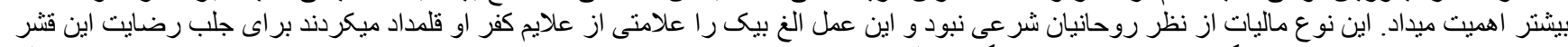

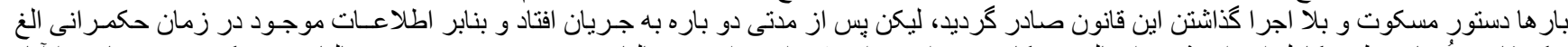

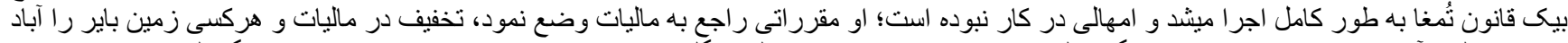

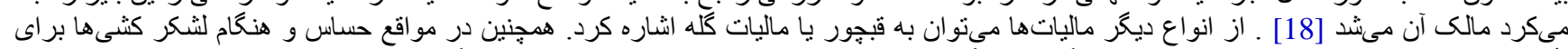

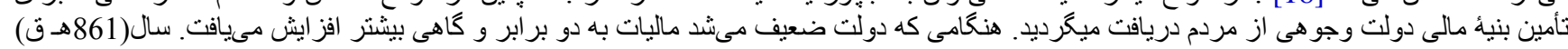

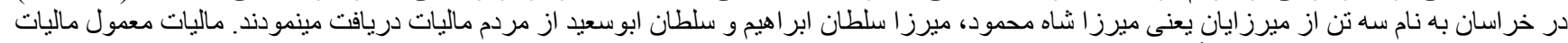

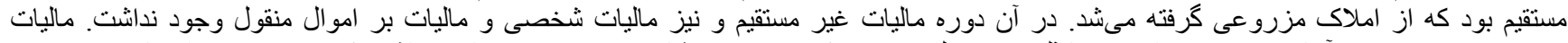

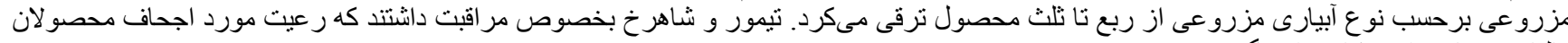

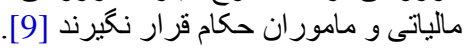

11.

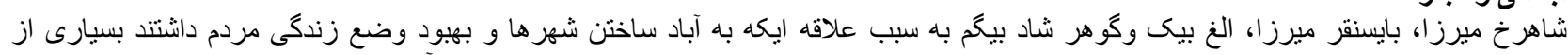

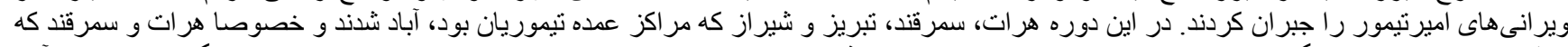

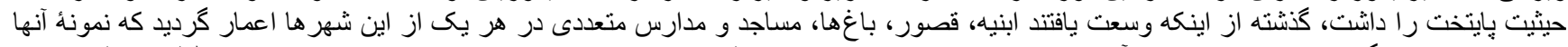

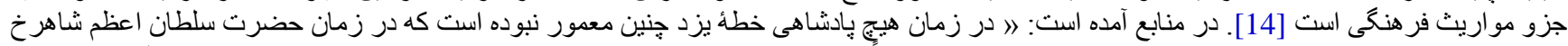

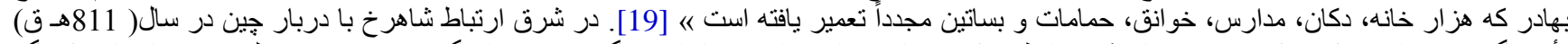

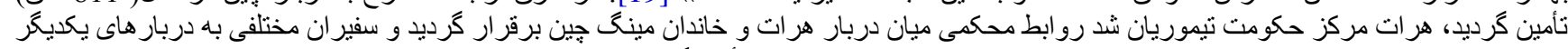

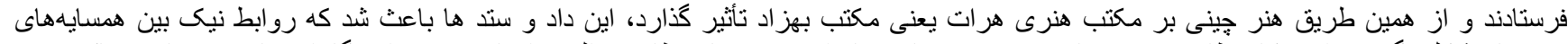

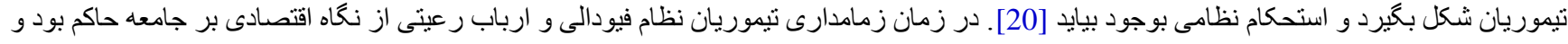




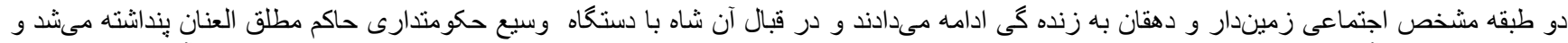

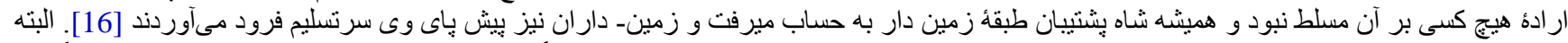

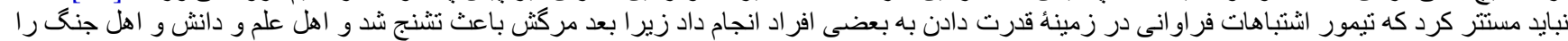

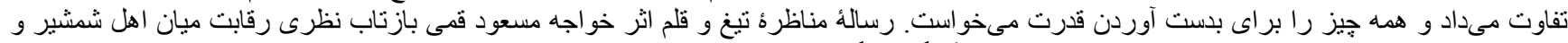

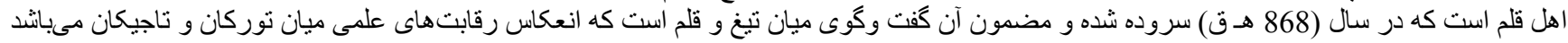

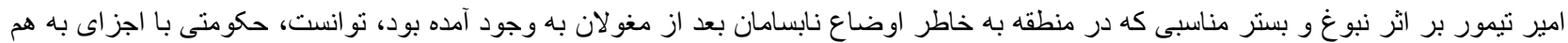

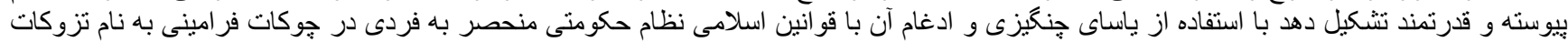

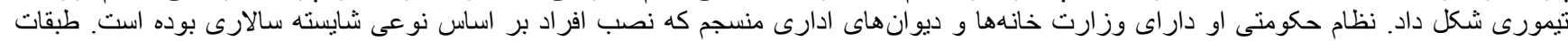

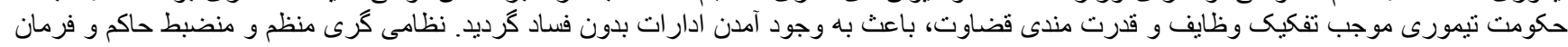

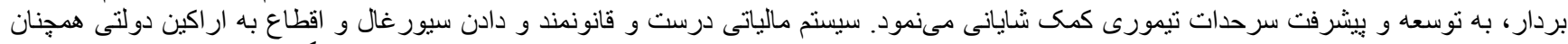

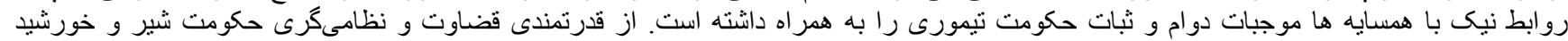

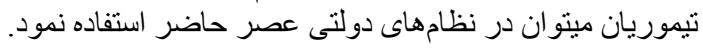

\section{References}

[1] Abdolhossein, Historical documents and correspondence of Iran from Timur to Shah Ismail. Tehran: Scientific and Cultural Publications, 1991.

[2] A. Mohammad, Herat in the Timurid era. Herat: Ahrari Publications, 2011.

[3] Abolfazl, The political and social situation of Iran in the eighth century AH from the fall of the Ilkhanids to the formation of the Timurids, 2nd ed.: Ferdowsi University of Mashhad, 2005.

[4] Clavijo, Clavijo's travelogue. Translated by Massoud Rajabnia. Tehran: Scientific and Cultural Publications, 1374.

[5] Ashtiani, The rise of Timur, by Mir Hashem Mohaddes. Tehran: National Works Association Publications, 1981.

[6] H. S. Mirjafari, Political, social, economic developments. And Iranian culture in the period Teymourians and Turkmens. Isfahan: Samat Publication, 2009.

[7] D. Samarkandi, Tazkereh Shoara. By Edward Brown. The Netherlands: Leiden Publishing, 1318.

[8] Baqer, "Teymourian civilization," Journal of the Faculty of Literature and Humanities, pp. 65-80, 1343.

[9] Khwandmir, History of Habib Al-Seer. Volume 3, by Mohammad Dabir Siyaghi and Homayi. Tehran: Khayyam Publications, 1333.

[10] Hosseini, Teymukat Teymouri. Tehran: Asadi Publications, 1342.

[11] Tabibi, History of Herat in the Timurid era. Tehran: Helmand Publications, 1989.

[12] Samarkandi, Wonders of the possible. The amazing life of Timur. Translated by Mohammad Ali Nejati. Tehran: Fars Publications, 1356.

[13] W. P. Lady, Biography and works of Amir Ali Shir Navai. Tehran: Al-Huda Publications, 2008.

[14] Yarshater, Persian poetry in the era of Shahrokh. Tehran: Tehran University Press, 1383.

[15] Brion, I am Timur Jahangsha, translated by Zabihullah Mansouri. Tehran: Mostofi Library Publications, 1372.

[16] M. Farahani, The link between politics and culture in the era of the decline of the Timurids and the rise of the Safavids. Tehran: University of Tehran Press, 2002.

[17] Ghobar, Afghanistan on the path of history. Kabul: Kabul Government Printing House, 1346.

[18] Barthold, History of the unseen and his time. Translated by Hossein Ahmadipour. Tabriz: Chehreh Publications, 1336.

[19] a.-K. Ali, The new history of Yazd. Tehran: Yazd Publications, 1317.

[20] Mehdi, History of Iranian Industries after Islam, translated by Mohammad Ali Khalili. Tehran: Jam Publications, 1320.

[21] S. Qomi, Debate and Pen Debate, by Seyyed Ali Al-Davood. Tehran: Toos Publications, 1997. 